\title{
Artigo/Article
}

\section{Doença de Chagas no Estado do Maranhão, Brasil: registro de casos agudos no período de 1994 a 2008}

\author{
Chagas' disease in the State of Maranhão, Brazil: record of acute cases from 1994 to 2008
}

\author{
Flávia Stella Rego Furtado Cutrim ${ }^{1}$, Irandir Augusto Almeida ${ }^{2}$, Eloísa da Graça do Rosário Gonçalves ${ }^{3}$ \\ e Antonio Rafael da Silva ${ }^{3}$
}

\begin{abstract}
RESUMO
Introdução: No Estado do Maranhão, a doença de Chagas não apresenta o padrão clássico de transmissão endêmica. No entanto, inquérito entomológico prévio constata altos índices de infecção natural dos vetores e casos agudos têm sido registrados nas últimas décadas, o que motivou o presente estudo que tem por objetivo avaliar as condições sociodemográficas e ambientais envolvidas na transmissão. Métodos: Foram estudados os casos agudos de doença de Chagas de 1994 a 2008. Os dados relacionados aos pacientes foram obtidos do banco de dados do Sistema de Informação de Agravos de Notificação, dos livros de registros de endemias da Fundação Nacional de Saúde e de prontuários médicos. Investigação entomológica foi realizada a partir de 2002. Resultados: Foram identificados 32 casos, procedentes de 17 municípios, sendo 84,4\%, da zona rural. O sexo masculino foi acometido em $67 \%$ dos casos. A ocupação mais frequente foi a de estudante $(38,9 \%)$, seguida pela de lavrador e de caçador (27,8\%). Conclusões: Os dados analisados sugerem que a transmissão foi, predominantemente, vetorial nos ambientes silvestre e peridomiciliar.
\end{abstract}

Palavras-chaves: Doença de Chagas. Estado do Maranhão.

\begin{abstract}
Introduction: Chagas' disease is not considered an endemic disease in the State of Maranhão. However, entomological surveys showed high natural vector infection indices and acute cases have been identified in the last few decades. This study aimed to analyze the social, demographic and environmental conditions involved in transmission. Methods: The study describes acute Chagas' disease identified from 1994 to 2008. Information regarding the cases was obtained from the Information System for Notifiable Diseases (Sinan), National Health Foundation (Funasa) database and medical records. Entomological surveys were conducted from 2002. Results: Data analysis indentified 32 cases from 17 municipal districts, with $84.4 \%$ of patients from rural areas. The disease was more frequent in men (67\%). The most frequent occupation was student, $38.9 \%$ of cases, followed by farmer and hunter, $27.8 \%$. Conclusions: The study suggests that transmission was vectorial and occurred in the wild or peridomicile.
\end{abstract}

Key-words: Chagas' disease. State of Maranhão.

\footnotetext{
1. Programa de Pós Graduação em Saúde e Ambiente, Universidade Federal do Maranhão, São Luís, MA 2. Coordenação do Programa de Controle de Doença de Chagas do Estado do Maranhão, São Luís, MA 3. Centro de Referência em Doenças Infecciosas e Parasitárias, Departamento de Patologia, Universidade Federal do Maranhão, São Luís, MA.

Endereço para correspondência: Dra. Eloísa da Graça do Rosário Gonçalves. Dept ${ }^{\circ}$ de Patologia/UFMA. Praça Madre Deus n² 2/Térreo, Bairro Madre Deus, 65025-560 São Luis, MA.

Tel: 5598 3221-0270

e-mail: credip@ufma.br

Recebido para publicação em $01 / 05 / 2010$

Aceito em 01/09/2010
}

\section{INTRODUÇÃO}

A Organização Mundial da Saúde (OMS) indicava a existência de 16-18 milhões de infectados pelo Trypanosoma cruzi nas Américas e de outros 90 milhões expostos ao risco de contrair a infecção, em $2005^{1}$. Em publicação mais recente são estimados de 12-14 milhões de indivíduos infectados na América Latina ${ }^{2}$. A inexistência de inquérito epidemiológico recente indica a necessidade de conhecimento da prevalência e incidência mais precisas da doença. De qualquer modo, os números ainda indicam a importância social da doença de Chagas, 100 anos após a descrição do primeiro caso agudo no Brasil ${ }^{3}$.

No Estado do Maranhão, os primeiros casos autóctones foram descritos em 1975, sendo três provenientes da Ilha de São Luis e um da Baixada Maranhense ${ }^{4}$. Até então, a doença era considerada inexistente no estado, onde a dinâmica de transmissão do Trypanosoma cruzi ainda não é totalmente compreendida, na medida em que apresenta peculiaridades regionais, que interferem na interação deste parasita com seus hospedeiros e vetores. Em inquérito sorológico nacional, aplicado entre 1975 e 1980, como parte do Programa de Controle da Doença de Chagas, do Ministério da Saúde, foi constatada, no Estado do Maranhão, uma prevalência de infecção de $0,1 \%$. Já em levantamento entomológico, realizado em 1996, foram capturados 290.576 exemplares de triatomíneos no país ${ }^{6}$. Destes, 201.156 foram na região Nordeste, sendo 1.240 no Estado do Maranhão. Enquanto no Brasil, em torno de 1\% dos barbeiros apresentou-se infectados por Trypanosoma cruzi, no Estado do Maranhão esta proporção chegou a $35,8 \%$ dos exemplares capturados ${ }^{6}$. A observação desses altos índices de infecção natural dos vetores motivou o desenvolvimento do presente estudo, que busca avaliar as condições sociodemográficas e ambientais envolvidos no surgimento da doença aguda, nos últimos anos, em um estado considerado não endêmico para a doença. 


\section{MÉTODOS}

Foram incluídos na análise os casos diagnosticados como agudos, pela presença do parasita em sangue periférico (exame a fresco ou gota espessa), segundo padronização do Manual de Doença de Chagas Aguda ${ }^{7}$. Dados demográficos, epidemiológicos e clínicos dos pacientes foram obtidos a partir dos livros de registros de casos da Fundação Nacional de Saúde (FNS), iniciados em 1994 e que constam de nome e procedência; do Sistema de Informação de Agravos de Notificação (SINAN), a partir de 2002 que, além dos dados anteriores, apresentam informação sobre a idade, a ocupação e as características do domicílio. A caracterização clínica se deu a partir dos dados registrados em prontuários médicos. Investigação entomológica nos municípios de origem dos pacientes foi realizada a partir de 2002 .

\section{RESULTADOS}

Foram notificados 32 casos agudos de doença de Chagas de 1994 a 2008, dentre os quais 18 constam dos registros do SINAN. Houve registro de casos em municípios localizados nas regiões noroeste, nordeste, central e sudoeste do estado. Destaca-se que 5 (15,6\%) casos foram oriundos de zona urbana e 27 (84.4\%), de zona rural. Os anos de 1997 e 2008 apresentaram maior número de notificações, com seis e cinco casos respectivamente, sendo que nos anos de 1999 e 2000 não houve notificação. A maioria dos municípios acometidos apresentou apenas um caso da doença. O Município de Turiaçu registrou cinco casos (Tabela $\mathbf{1}$ ).

TABELA 1 - Distribuição espaço-temporal dos casos de doença de Chagas aguda registrados no Estado do Maranhão, 1994-2008.

\begin{tabular}{|c|c|c|c|c|}
\hline \multirow[b]{2}{*}{ Período de estudo } & \multicolumn{2}{|c|}{ Casos } & \multirow{2}{*}{$\begin{array}{l}\text { Número de municípios } \\
\text { acometidos no período }\end{array}$} & \multirow[b]{2}{*}{ Número de casos/município } \\
\hline & $\mathrm{n}^{\mathrm{o}}$ & $\%$ & & \\
\hline \multirow[t]{2}{*}{ 1994-1996 } & 4 & 12,5 & 2 & 3/São Luis \\
\hline & & & 1/São Bento & \\
\hline \multirow[t]{4}{*}{ 1997-1999 } & 8 & 25,0 & 4 & 4/Raposa \\
\hline & & & 2/Itapecuru & \\
\hline & & & 1/Tutóia & \\
\hline & & & 1/Icatu & \\
\hline \multirow[t]{4}{*}{ 2000-2002 } & 5 & 15,6 & 4 & 1/São Luís \\
\hline & & & 1/Viana & \\
\hline & & & 2/Penalva & \\
\hline & & & 1/Turiaçu & \\
\hline \multirow[t]{5}{*}{ 2003-2005 } & 7 & 21,9 & 5 & 2/Amarante do Maranhão \\
\hline & & & $1 /$ Esperantinópolis & \\
\hline & & & 1/Turiaçu & \\
\hline & & & 1/Miranda do Norte & \\
\hline & & & 2/Presidente Vargas & \\
\hline \multirow[t]{6}{*}{ 2006-2008 } & 8 & 25,0 & 6 & 1/Buriticupu \\
\hline & & & 1/Itinga & \\
\hline & & & 3/Turiaçu & \\
\hline & & & 1/Icatú & \\
\hline & & & 1/Presidente Sarney & \\
\hline & & & 1/Axixá & \\
\hline Total & 32 & 100,0 & 17 & $32 / 17$ \\
\hline
\end{tabular}

\section{Caracterização sociodemográfica dos pacientes}

Para esta análise, foram considerados os 18 pacientes, cujos registros constam do SINAN, a partir de 2002. Doze (67\%) eram do sexo masculino e 6 (33\%), do feminino. Destaca-se que houve maior número de casos na faixa etária entre 0 e 20 anos de idade (Tabela 2).

TABELA 2 - Distribuição por faixa etária e sexo dos casos de doença de Chagas aguda, registrados no Estado do Maranhão, 2002-2008.

\begin{tabular}{lccc}
\hline Faixa etária & Masculino & Feminino & Percentagem (\%) \\
\hline $0-10$ & - & 4 & 22,2 \\
$11-20$ & 4 & - & 22,2 \\
$21-30$ & 1 & - & 5,6 \\
$31-40$ & 2 & 1 & 16,6 \\
$41-50$ & 1 & - & 5,6 \\
$51-60$ & 2 & - & 11,0 \\
$61-70$ & 1 & - & 5,6 \\
$71-80$ & 1 & - & 5,6 \\
$81-90$ & - & 1 & 5,6 \\
\hline Total & $\mathbf{1 2 ( 6 6 , 7 \% )}$ & $\mathbf{6 ( 3 3 , 3 \% )}$ & $\mathbf{1 0 0 , 0}$ \\
\hline
\end{tabular}

A ocupação mais referida foi a de estudante com 7 (38,9\%) casos, seguida de lavrador/caçador com $5(27,8 \%)$, pescador com 2 (11\%), trabalhador de serraria $1(5,5 \%)$ e aposentado $1(5,5 \%)$. Em 2 (16,6\%), casos não havia registro da atividade profissional.

Foi possível avaliar as condições de moradia de treze pacientes. Os domicílios, estruturalmente, possuíam paredes de taipa rebocadas com barro em 9 (50\%); de alvenaria em 2 (11\%) e de tábuas em 2 (11\%). O piso era de chão batido em 10 (55\%) e de cimento em 3 (17\%) deles. A cobertura era feita de telha tipo amianto ou cerâmica em 9 (50\%) e de palha em 4 (22\%). Só 3 (17\%) possuíam água encanada. Água tratada, esgoto e energia elétrica estavam presentes em 4 (22\%) domicílios, enquanto 9 (50\%) não os possuíam.

\section{Manifestações clínicas}

As manifestações clínicas relatadas nas fichas de investigação e nos prontuários médicos estão apresentadas na Tabela 3.

\section{Estudo entomológico}

Nas investigações entomológicas, feitas em relação aos casos ocorridos a partir de 2002, foram encontrados triatomíneos no peridomicílio em duas ocasiões: em novembro de 2005, no Município de Presidente Vargas, em duas palmeiras distantes cerca de $10 \mathrm{~m}$ da casa

TABELA 3 - Manifestações clínicas dos casos de doença de Chagas aguda registrados no Estado do Maranhão, 2002-2008.

\begin{tabular}{lcc}
\hline Sinais e sintomas & Número & Porcentagem (\%) \\
\hline Chagoma de inoculação & 2 & 11,2 \\
Hepatoesplenomegalia & 3 & 16,8 \\
Insuficiência cardíaca congestiva & 4 & 22,4 \\
Febre & 13 & 72,2 \\
Cefaléia & 8 & 44,4 \\
Adenopatia & 1 & 5,6 \\
Edema generalizado & 2 & 11,2 \\
Edema localizado & 2 & 11,2 \\
Estado geral comprometido & 12 & 66,6 \\
\hline
\end{tabular}


do paciente, nas quais foram encontrados quatro exemplares de Rhodnius robustus. Um exemplar estava positivo para as formas tripomastigotas metacíclicas de Trypanosoma cruzi. Em abril de 2008, no Município de Turiaçú, numa casa de farinha situada no peridomicílio próximo a um riacho, foram capturados oito exemplares da espécie Rhodnius pictipes. Tinha estrutura de esteios, com paredes de palha de aproximadamente $1 \mathrm{~m}$ de altura, teto de palha, com várias aberturas e rodeada de vegetação alta de várias espécies. Em visita posterior, em junho de 2008, a equipe encontrou mais um exemplar de Rhodnius pictipes no mesmo local.

\section{DISCUSSÃO}

O registro de casos agudos de doença de Chagas em quatro das seis regiões geográficas do Estado do Maranhão sinaliza o potencial da transmissão do Trypanosoma cruzi em paisagens fitogeográficas muito diversificadas, como floresta amazônica, floresta baixa, cerrados, palmeirais e região litorânea. Esta distribuição coincide com as áreas onde houve o maior número de triatomíneos capturados em inquéritos entomológicos realizados previamente ${ }^{8}$, destacando-se a procedência predominante de área rural dos municípios envolvidos.

O achado da maioria dos casos entre o sexo masculino (67\%), numa análise isolada, sugere transmissão silvestre, uma vez que são os homens que adentram na mata para caçar ou cuidar da lavoura, por dias consecutivos, expondo-se mais ao vetor, o que é corroborado pelas ocupações mais referidas entre os adultos que são lavrador e caçador. No entanto, quando se analisa a ocorrência da doença, por faixa etária, os resultados mostram o envolvimento de todas as faixas etárias, com maior prevalência entre 0 e 20 anos. Outros estudos mostraram o perfil da incidência da forma aguda em função da faixa etária com os maiores valores entre os mais jovens, configurando a correlação direta entre a incidência da doença e o tempo de vida em áreas nas quais o habitat do principal vetor é intradomiciliar. Curvas com esse desenho foram observadas em estudos realizados em áreas como Iguatama ${ }^{9}$, Berilo $^{10}$ e Virgem da Lapa ${ }^{11}$ em Minas Gerais, todas com transmissão vetorial interrompida há, pelo menos, 20 anos. O conhecimento da idade é relevante, pois, a morbimortalidade da doença de Chagas aguda guarda, em geral, relação direta com a idade do paciente. Neste estudo, um (5,5\%) caso evoluiu para óbito na primeira faixa etária (0-10 anos).

A ocorrência de parcela significativa dos casos em menores de idade poderia ser explicado pelo fato dos pais, ao realizarem suas atividades de fabricação de farinha nas casas de forno, levarem seus filhos para acompanhar o processo que pode durar até uma semana. $\mathrm{Ou}$, ainda, pode-se pensar em introdução do vetor no domicílio, atraído pela luz e por animais domésticos. A propósito, estudo sobre o comportamento dos vetores silvestres no município de Paço do Lumiar, Estado do Maranhão, concluiu que eles invadem as casas, durante a noite, nas estações chuvosas, atraídas pela luz e pelo alimento ${ }^{12}$. O contato direto ou indireto com triatomíneos pode ocorrer sem evidência marcante, justamente em virtude desse comportamento de insetos não domiciliados, que eventualmente adentram os domicílios humanos ${ }^{10,13,14}$.

O estudo sugere que, no Estado do Maranhão, a infecção por Trypanosoma cruzi se dê, predominantemente, no ambiente silvestre. Um fator que indica a domiciliação do vetor é a presença da ninfa no domicílio. Nas capturas realizadas, no presente estudo, não foram encontradas ninfas do barbeiro no interior das casas, sendo encontrados vetores adultos no peridomicílio em apenas dois casos. No entanto, a investigação entomológica ocorreu semanas após as notificações, fato que não prova ou não permite concluir o local exato da transmissão. Nenhuma generalização pode ser feita a partir de um único estudo enzoótico de parasitas, pois cada ecótopo deve ser considerado único ${ }^{15}$. Por isso, as ações de controle e de certificação devem ser avaliadas de forma clara em cada área geográfica. A associação da doença de Chagas com péssimas condições socioeconômicas tem sido referida em todos os estudos epidemiológicos realizados em áreas rurais da América Latina ${ }^{16}$. A caracterização dos domicílios dos casos registrados no Maranhão reflete as condições precárias em que vive a população das áreas rurais nas quais a doença foi predominante.

Os resultados das manifestações clínicas dos pacientes incluídos neste estudo mostraram concordância com a literatura ${ }^{13}$. A maioria dos sinais e sintomas são inespecíficos e constituem elementos para os frequentes equívocos diagnósticos com algumas endemias prevalentes na região, especialmente malária e dengue, sendo o sinal mais frequente a febre ${ }^{17}$. Na casuística estudada, houve, da mesma forma, predomínio do quadro febril nas manifestações clínicas descritas, concordando com estudos realizados em áreas endêmicas.

\section{CONFLITO DE INTERESSE}

Os autores declaram não haver nenhum tipo de conflito de interesse no desenvolvimento do estudo.

\section{SUPORTE FINANCEIRO}

Coordenação de Aperfeiçoamento de Pessoal de Nível Superior (CAPES) e Fundação de Amparo à Pesquisa e ao Desenvolvimento Científico e Tecnológico do Maranhão (FAPEMA).

\section{REFERÊNCIAS}

1. World Health Organization. Control of Chagas' disease. Technical Report Series 2005; 905:96.

2. Villela MM, Souza JMB, Melo VP, Dias JCP. Vigilância epidemiológica da doença de Chagas em programa descentralizado: avaliação de conhecimentos e práticas de agentes municipais em região endêmica de Minas Gerais, Brasil. Cad Saude Publica 2007; 23:2428-2438.

3. Chagas C. Trypanosomíase americana: forma aguda da moléstia. Mem Inst Oswaldo Cruz. 1916; 8:37-60.

4. Silva AR, Mendes JRB, Mendonça ML, Cutrim RN, Brasil RP. Primeiros casos agudos autóctones da doença de Chagas no Maranhão e inquérito soroepidemiológico da população. Rev Soc Bras Med Trop 1985; 18:269-270.

5. Camargo ME, Silva GR, Castilho EA, Silveira AC. Inquérito sorológico da prevalência da infecção chagásica no Brasil, 1975 a 1980. Rev Inst Med Trop de São Paulo 1984: 26:192-204.

6. Ministério da Saúde. Fundação Nacional de Saúde. Controle da doença de Chagas. Diretrizes Técnicas. Brasília DF; 1994.

7. Ministério da Saúde. Secretaria Nacional de Vigilância em Saúde. Manual de doença de Chagas aguda. Brasília DF; 2004.

8. RebêloJMM. Bioecologia dos triatomíneos vetores da doença de Chagas. Manual para técnicos e profissionais da área de saúde. Universidade Federal do Maranhão, São Luis; 2000.

9. Coura JR, Junqueira ACV, Giordano CM, Funatsu IRK. Chagas disease in the Brazilian Amazon. I - A short review. Rev Inst Med Trop de São Paulo 1994; 36:363-368. 
10. Araújo RM. Morbidade da doença de Chagas no município de Berilo, Minas Gerais: estudo seccional e evolutivo de 10 anos (1987-1997). [Tese de Doutorado]. [Rio de Janeiro]: Instituto Oswaldo Cruz; 1998.

11. Borges-Pereira J. Doença de Chagas humana: estudo da infecção crônica, morbidade e mortalidade em Virgem da Lapa, MG, Brasil (1976-1996). [Tese de Doutorado]. [Rio de Janeiro]: Instituto Oswaldo Cruz; 1997.

12. Monteiro PS. Dinâmica de transmissäo de protozoários cinetoplastidas para a populaçäo humana de Paço do Lumiar, MA: descriçäo de rede trófica associada com a transmissão de Trypanosoma cruzi. [Tese de Doutorado]. [Brasília]: Faculdade de Ciências da Saúde, Universidade de Brasília; 2000.

13. Coura JR, Abreu LL, Dubois LE, Lima FC, Arruda-Jr ER, Willcox HP, et al. Morbidade da doença de Chagas. II - Estudos. Rev Soc Bras Med Trop 2006; 39:530-539.

14. Coura JR. Chagas's disease as endemic to the Brazilian Amazon: risk or hypothesis? Rev Soc Bras Med Trop 1990; 23:67-70.

15. Pinto AYN, Valente SA, Valente VC, Ferreira Jr AG, Coura JR. Fase aguda da doença de Chagas na Amazônia brasileira: Estudo de 233 casos do Pará, Amapá e Maranhão observados entre 1988 e 2005. Rev Soc Bras Med Trop 2008; 41:602-614.

16. Dias JCP. A Doença de Chagas e seu Controle na América Latina. Uma análise de possibilidades. Cad Saude Publica 1993; 9:201-209.

17. Crescente JA, Valente SAS, Valente VC, Araújo JEA. Ocorrência de quatro casos agudos de Doença de Chagas na vila de Icoaraci-PA. Rev Soc Bras Med Trop $1992 ; 25: 67$. 